\title{
The spacing effect in free recall emerges with development
}

\author{
THOMAS C. TOPPINO and WILLIAM DiGEORGE \\ Villanova University, Villanova, Pennsylvania
}

\begin{abstract}
In two experiments, children were shown a series of pictures of common objects and were asked to free recall which pictured objects had been presented. Some pictures were presented twice, with repetitions being either massed or spaced apart. Results indicated that preschoolers benefited from repetition but that they recalled massed and spaced repetitions equally well. In contrast, first-graders produced the typical spacing effect by recalling spaced repetitions better than they did massed repetitions. This finding that the spacing effect in free recall emerges with development suggests that the phenomenon cannot be explained solely in terms of a primitive encoding mechanism that is hard-wired into the memory system. Rather, an adequate account must include processes that are acquired or that develop during childhood. Further discussion focuses on implications of our findings for specific theories of the spacing effect and for the direction of future research.
\end{abstract}

That repetition improves memory is one of the most widely accepted generalizations that we have about learning and memory. Repetition seems to underlie many common learning strategies and is incorporated into many teaching techniques. It is commonly exploited in attempts at persuasion. Indeed, there is evidence that repetition increases the credibility of plausible statements (Hasher, Goldstein, \& Toppino, 1977)-a phenomenon that may be mediated by the memorability of repeated events (Bacon, 1979). Qualifying the generalization that repetition facilitates memory, however, is the fact that its effectiveness depends critically on the spacing between repetitions. When repetitions are presented in immediate succession (massed presentations), their beneficial effect is often minimal. However, as repetitions become more distributed or spaced apart, memory for repeated information typically improves.

The latter phenomenon is known as the spacing effect and has proved to be an extraordinarily robust finding. It has been obtained with a wide variety of materials ranging from nonsense syllables and words to picture and prose passages (Kraft \& Jenkins, 1981). It has been demonstrated with virtually every standard memory task from free recall to recognition (Hintzman, 1974), and occurs despite variation in task parameters such as rate and modality of presentation (e.g., Melton, 1970). The phenomenon is obtained in incidental learning conditions (e.g., Maskarinec \& Thompson, 1976; Shaughnessy, 1976), as well as in intentional learning

We would like to thank Jane Flamish for her assistance in preparing the stimuli and Pamela Blewitt for her helpful comments on an earlier draft of this paper. Reprint requests should be sent to Thomas C. Toppino, Department of Psychology, Villanova University, Villanova, Pennsylvania 19085. conditions (e.g., Hintzman \& Rogers, 1973; Melton, 1967; Underwood, 1969). And, the effect often has persisted despite attempts to eliminate or attenuate it (e.g., Bird, Nicholson, \& Ringer, 1978; Hintzman, Summers, Eki, \& Moore, 1975; Jensen \& Freund, 1981; Shaughnessy, 1976).

The uncommon generality and persistence of the spacing effect has led some investigators to propose that the phenomenon reflects the automatic operation of some fundamental memory process (e.g., Jensen \& Freund, 1981) that may even be hard-wired into the memory system (e.g., Hintzman, 1974; Landauer, 1969; Peterson, 1966). This hypothesis received support from a recent study by Cornell (1980), in which 5- and 6-month-old infants appeared to exhibit a spacing effect. Babies viewed human faces with repetitions spaced either $3 \mathrm{sec}$ or $1 \mathrm{~min}$ apart. After a retention interval that ranged from $5 \mathrm{sec}$ to $1 \mathrm{~h}$, a recognition test was administered in which infants were exposed simultaneously to an old and a new face and in which a preference for viewing the new face was interpreted as evidence for recognition. Results indicated that, for the longer retention intervals, the infants showed a preference for the new face following spaced repetitions but not following massed repetitions.

Although very fundamental-even primitive-memory processes must have been involved for Cornell (1980) to have obtained a spaced-repetition effect in infants, we were concerned about the degree to which this conclusion could be generalized to other situations. First, infant recognition procedures depending on differential viewing time for two stimuli are drastically different from the procedures used to study conscious episodic memory with older children and adults. There is no guarantee that the two kinds of procedure tap into the same processes (Werner \& Perlmutter, 1979). Second, it 
has been suggested that different information may underlie repetition and spaced-repetition effects in recognition tasks and other tasks such as free recall. For example, according to Glenberg (1979; Glenberg \& Smith, 1981), recognition depends primarily on the encoding of characteristic information that distinguishes one to-be-remembered item from another, whereas free recall depends more heavily on the encoding of contextual information. Thus, even if we extrapolate from Cornell's findings and conclude that primitive mech. anisms are sufficient to produce a spacing effect in the recognition memory of older children and adults, it does not necessarily follow that this is true of the spacing effect in general, because the mechanisms may not operate automatically on the information that mediates performance in other memory tasks.

To explore this issue further, we decided to investigate the spacing effect in free recall and to do so with a population of preschool children who seem to possess the following characteristics. First, they are old enough to perform conscious, episodic memory tasks such as those employed with adults. And, second, they are so young that they are unlikely to use intentional memorization strategies as adults do (Myers \& Perlmutter, 1978; Paris, 1978). Rather, remembering for them seems to be the result of more fundamental memory processes.

\section{EXPERIMENT 1}

\section{Method}

Subjects and Design. The subjects were 20 children from suburban nursery schools [mean age $=4$ years 4 months $(4-4)$; range $=4-0$ to $5-0]$. They participated in a single-factor experiment in which the spacing between repetitions (massed vs. spaced) was manipulated as a within-subjects variable.

Materials. Four 22-position lists were developed, two using each of two different list structures constructed with the same set of general constraints, as follows. To control for serial position effects during free recall, the first and last 3 serial positions were reserved for once-presented items that served as primacy and recency buffers, respectively. The middle portions of the list structures consisted of 16 positions that were designated for the presentation of four once-presented filler items and six twice-presented items. Of the latter items, three were massed repetitions (separated by no other items) and three were spaced repetitions (separated by the presentation of three other items). To control further for possible extended recency effects (e.g. Underwood, 1969), massed and spaced repetitions were located in the list structures such that they were equated with respect to the mean serial positions of their second occurrences.

The four actual experimental lists were constructed from a pool of 16 pictures of unrelated common objects that were selected because they could be labeled easily and unambiguously by preschool children. To create the first list involving each list structure, pictures were assigned to serve as primacy buffers, recency buffers, fillers, massed repetitions, and spaced repetitions. They were then assigned to serial positions in the list structures. The second list involving each list structure was composed by interchanging the pictures that served as massed and as spaced repetitions, respectively. Thus, across lists, the same set of items served equally often in each repetition condition.

Procedure. The children participated individually. Five children received each of the four lists. The experimenter presented the sequence of pictures manually at a $5-\sec$ rate. As each
Table 1

Mean Percent Correct Recall in Two Experiments as a Function of Grade Level and Presentation Condition

\begin{tabular}{lccc}
\hline & $\begin{array}{c}\text { Single } \\
\text { Presentation }\end{array}$ & $\begin{array}{c}\text { Massed } \\
\text { Repetitions }\end{array}$ & $\begin{array}{c}\text { Spaced } \\
\text { Repetitions }\end{array}$ \\
\hline Preschoolers & Experiment 1 & & \\
& 11.2 & 46.7 & 46.7 \\
& Experiment 2 & & \\
Preschoolers & 13.9 & 34.7 & 32.0 \\
First Graders & 20.8 & 37.5 & 54.2 \\
\hline
\end{tabular}

picture was presented, the children were required to label it. Following list presentation, the children were allowed $3 \mathrm{~min}$ for oral free recall.

\section{Results and Discussion}

The mean percentages of correct recall of repeated items are presented in Table 1 . Clearly, the spacing between repetitions had no effect on performance. However, there were several reasons for exercising caution in interpreting these results.

First, accepting an unreplicated null effect is always risky, and this is especially so when it involves the failure to obtain a phenomenon that is as general and robust as the spacing effect. Second, a skeptic might question whether repetition had affected performance at all in Experiment 1. Even though the children recalled only $11.2 \%$ of the once-presented items in the middle region of the list (in comparison with $46.7 \%$ for repeated items), we had made no effort to equate once-presented and repeated items in terms of item difficulty or serial position. It could be argued that either or both of these factors could have accounted for the apparent repetition effect that we obtained. Third, as far as we know, no other spaced-repetition study had ever used the procedures that we employed (i.e., recall of pictures). Thus, before we took seriously the failure to obtain a spacing effect with these procedures in one population, it was important to demonstrate that the procedures did result in a spacing effect in another, older population. Experiment 2 was designed with the foregoing points in mind.

\section{EXPERIMENT 2}

\section{Method}

Subjects and Design. The subjects were 18 nursery school children (mean age $=4-4 ;$ range $=3-11$ to $5-2$ ) and 18 first-grade children (mean age $=6-8$; range 6-5 to $7-4$ ). In addition to the grade-level factor, presentation condition (once-presented, massed repetition, and spaced repetition) was manipulated within subjects.

Procedure and Materials. The procedure was identical to that used in Experiment 1. With regard to materials, list structures and lists were constructed in the same manner as those in Experiment 1 . The only differences were as follows. Lists contained 27 serial positions. The first 3 and last 4 positions were reserved for primacy and recency buffers, respectively. The middle portion of the list structures contained four once-presented items, 
four massed repetitions, and four spaced repetitions. Two items from each of these three conditions occurred in the first half of the list, whereas the remaining two items from each condition occurred in the second half of the list. The mean serial position of once-presented items was equated with that of the second occurrences of massed and spaced repetitions.

Six lists, three for each of two list structures, were constructed from a pool of 19 pictures of common unrelated objects. So that the same set of items would serve in all three repetition conditions (i.e., once-presented, massed repetition, and spaced repetition), a Latin-square principle was used to counterbalance the pictures serving in these conditions across the three lists involving each list structure. Three children from each grade level received each of the six lists.

\section{Results and Discussion}

The mean percentages of correct recall are presented in Table 1. A series of planned comparisons were conducted on the data. For preschool children, repeated items were recalled better than once-presented items $[F(1,34)=10.02, p<.01]$, but there was no difference between recall of massed and spaced repetitions $[F(1,34)$ $<1.00$ ] ( $\mathrm{MSe}=452.75$ in both cases). Thus, in a situation in which preschoolers clearly benefited from repetition, we were able to replicate our previous finding that they do not exhibit a spacing effect in free recall. We were also able to demonstrate that exactly the same procedures produced a spacing effect in first-grade children. These children not only recalled repeated iterns better than they did once-presented items $[F(1,34)=$ $22.67, \mathrm{p}<.01]$, but, more importantly, they also recalled spaced repetitions better than they did massed repetitions $[F(1,34)=7.56, p<.01](\mathrm{MSe}=330.88$ in both cases). ${ }^{1}$

\section{GENERAL DISCUSSION}

Our fundamental findings were that preschoolers do not exhibit a spacing effect in free recall, although they do benefit from repetition. With further development, however, the spacing effect does emerge. These findings place constraints on the hypothesis that the spacing effect is due to a mechanism that is hard-wired into the memory system and that operates automatically. If such a mechanism exists, as suggested by Cornell's (1980) data, its field of operation may be limited to recognition memory. The spacing effect in free recall appears to depend upon the development of other (but perhaps analogous) mechanisms. At the very least, if a primitive mechanism does underlie the spacing effect in free recall, it is apparently not sufficient to produce the phenomenon until it has been augmented by the development of other processes.

With regard to the implications of our findings for specific theories of the spacing effect, it is convenient to consider two major categories of theory: deficientprocessing theories and encoding variability theories. According to the former type of theory, poor memory for massed repetitions is explained by assuming that one presentation or the other is not fully processed. With increased spacing, the shortchanged presentation receives more processing, and thus memory improves. According to the latter kind of theory, repetition facilitates memory to the extent that each presentation is encoded differently, thereby establishing more ways to access the information at retrieval. When repetitions are massed, both presentations are likely to be encoded similarly. However, as spacing between repetitions increases, encodings become increasingly different, and this produces better memory.

Among the major deficient-processing theories, the one that seems most compatible with our results proposes that subjects adopt a voluntary strategy in which they do not attend to and fully process the second occurrence of a massed repetition, but in which they devote more attention to the second occurrence as spacing increases. To account for our findings, one might assume that this strategy develops or is acquired between the ages of approximately 4 and 6 years. Although it is not at all clear why such a strategy would be acquired at this point in development, it is at least plausible. Research with tasks involving selective attention such as memory for central versus incidental information (e.g., Hagen \& Stanovich, 1977), concept identification (e.g., Johnson, Warner, \& Silleroy, 1971; Toppino, Lee, Johnson, \& Shishko, 1979), and same-different judgments of multidimensional stimuli (e.g., Pick \& Frankel, 1974) has indicated that there is a general trend for attention to become increasingly "active and directed" (Pick, Christy, \& Frankel, 1972, p. 165) with development. The emergence of the strategy under consideration here may be another indication of this general developmental trend.

Other deficient-processing theories fare less well with our data. Two of these, consolidation theory and habituation theory, have not been convincingly confirmed or disconfirmed by previous research (e.g., Hintzman, 1974; Hintzman, Summers, \& Block, 1975). In light of the unusual generality and persistence of the spacing effect, these theories have remained somewhat attractive for the same reason that they cannot explain our find ings. That is, they attribute the spacing effect to involuntary processes associated with the basic neurophysiology of memory. According to the first theory, deficient processing occurs because consolidation of one presentation interferes with consolidation of the other when repetitions are too close together. According to the second theory, encoding one presentation temporarily habituates some encoding process such that it cannot properly respond to a second presentation until sufficient time has passed for recovery to have taken place. To account for our results, these theories would have to make the implausible assumption that the time course of consolidation or habituation-recovery gets substantially longer between the ages of 4 and 6 years

A final deficient-processing theory is based on the assumption that massed repetitions receive less total rehearsal than do spaced repetitions. Although this 
theory has difficulty with other findings (see Hintzman, 1974 , for a review), it is discussed here because, at first glance, it seems able to account for our data. That is, research on the development of rehearsal indicates that preschoolers are unlikely to engage in spontaneous rehearsal, whereas first-graders may do so (e.g., Flavell, Beach, \& Chinsky, 1966; Keeney, Cannizzo, \& Flavell, 1967). Thus, if the spacing effect were based on rehearsal, it seems reasonable that preschoolers would not show an effect but that first-graders might. Unfortunately, this explanation does not hold up on closer analysis.

According to the theory, massed and spaced repetitions differ in how much rehearsal is given to the first presentation before the second presentation occurs (Rundus, 1971). It is assumed that an item is rehearsed not only at the time of its own presentation, but also during the presentation of subsequent items. Thus, there is more opportunity for rehearsal between repetitions for spaced than for massed repetitions. If an item were rehearsed only during its own presentation, two presentations would lead to the same amount of rehearsal regardless of the spacing between them. Therein lies the problem that this theory has with our findings. Although first-graders might exhibit spontaneous rehearsal, they would be expected to rehearse only one item at a time and only the most recently presented item (e.g., Ornstein, Naus, \& Liberty, 1975; Ornstein, Naus, \& Stone, 1977). Thus, if rehearsal underlies the spacing effect, we should have obtained no spacing effect for either preschool or first-grade children.

Now consider theories that can be classified as encoding variability theories (e.g., Bower, 1972; Glenberg, 1979; McFarland, Rhodes, \& Frey, 1979). These theories are often vague with regard to whether variable encoding is an involuntary result of basic memory mechanisms or whether it is the result of voluntary strategies. Regardless of what produces variable encoding, however, these theories suggest either implicitly or explicitly that the probability of successfully retrieving a trace of a repeated item equals the probability of retrieving information encoded on the first presentation, information encoded on the second presentation, or both. It is assumed that, with increasing spacing, the total probability of successful retrieval increases because the information encoded on the first and second presentations becomes increasingly independent (i.e., less overlapping).

Although different encoding variability theories vary in a number of details, they all seem compatible with the following general account of our results. The performance of first-graders is explained in the manner that has already been outlined. The performance of preschoolers, however, is explained by assuming that they encode every item presentation as a completely independent event. Thus, they would recall repeated items better than they would oncepresented items. But, because their encodings would be independent regardless of the spacing between repe- titions, spacing would not influence their performance.

The above hypothesis is attractive in its simplicity and apparent plausibility. However, there is also reason to question the adequacy of an account of our data based on encoding variability theories. These theories imply that repetition produces its maximum effect when the information encoded on each presentation is completely independent. This maximum level of performance can be estimated using the proportion $(\mathrm{P})$ of once-presented items correctly recalled as an estimate of the probability of successfully retrieving a single independent presentation. The estimated probability, then, of retrieving either or both of two independent presentations is given by $\mathrm{P}+\mathrm{P}-\mathrm{P}^{2}$, or $2 \mathrm{P}-\mathrm{P}^{2}$. In Experiment 2, preschool children recalled .139 of the oncepresented items. Thus, the estimated probability of recalling one or both of two independent events was .259 , which was reliably lower than the obtained level of recall $(.333)$ for repeated items $[t(17)=2.31, p<.05] .^{2}$ In short, preschoolers recalled repeated items significantly better than should have been possible according to encoding variability theories. ${ }^{3}$

In summary and conclusion, our findings place limits on the kind of explanation that will provide an adequate general account of the spacing effect. Such an explanation cannot rely solely on automatic encoding processes that are operative from early infancy, but must incorporate processes that become active later in childhood. Of the major theories that have been offered to explain the spacing effect in adults, the one that seems most easily reconciled with our findings attributes the spacing effect to a strategy in which subjects voluntarily divert attentional processing from the second occurrence of a massed repetition. Further research will be necessary to determine whether the difference in performance between preschool and first-grade children is best explained by the acquisition of such an attentional strategy or by the development of some other process or set of processes. However, a more important point may be that our findings suggest that a new approach to the study of spaced-repetitions may be productive.

The last 15 years have witnessed a great number of experiments investigating the effects of spaced repetitions in human adults. Because adult subjects produce the spacing effect in most circumstances, the predominant research strategy for isolating the underlying mechanism(s) has involved finding conditions that eliminate or attenuate the effect. Unfortunately, this approach has not led to a generally accepted explanation of the spacing effect. Our findings suggest that it may be fruitful to take a nearly opposite, but complementary, approach-a developmental approach (see also Toppino \& DeMesquita, in press, and Wilson, 1976). We have found a population of children who do not yet exhibit the spacing effect in free recall. If future research can discover what developments or acquisitions lead to the emergence of the spacing effect in children, the findings should illuminate the mechanisms underlying the spacing effect in general. 


\section{REFERENCES}

Bacon, F. T. (1979). Credibility of repeated statements: memory for trivia. Journal of Experimental Psychology: Human Learning and Memory, 5, 241-252.

Bird, C. P., Nicholson, A. J., \& Ringer, S. (1978). Resistance of the spacing effect to variations in encoding. American Journal of Psychology, 91, 713-721.

Bower, G. H. (1972). Stimulus-sampling theory of encoding variability. In A. W. Melton \& E. Martin (Eds.), Coding processes in human memory. Washington, DC: V. H. Winston.

Corneli, E. H. (1980). Distributed study facilitates infants' delayed recognition memory. Memory \& Cognition, 8, 539-542.

Flavell, J. H., Beach, D. H., \& Chinsky, J. M. (1966). Spontaneous verbal rehearsal in a memory task as a function of age. Child Development, 37, 283-299.

Glenberg, A. M. (1979). Component-levels theory of the effects of spacing of repetitions on recall and recognition. Memory \& Cognition, 7, 95-112.

Glenbero, A. M., \& Sмith, S. M. (1981). Spacing repetition and solving problems are not the same. Journal of Verbal Learning and Verbal Behavior, 20, 110-119.

Hagen, J. W., \& Stanovich, K. G. (1977). Memory: Strategies of acquisition. In R. V. Kail, Jr., \& J. W. Hagen (Eds.), Perspectives on the development of memory and cognition. Hillsdale, NJ: Erlbaum.

Hasher, L., Goldstein, D., \& Toppino, T. (1977). Frequency and the conference of referential validity. Journal of Verbal Learning and Verbal Behavior, 16, 107-112.

Hintzman, D. L. (1974). Theoretical implications of the spacing effect. In R. L. Solso (Ed.), Theories in cognitive psychology: The Loyola Symposium. Hillsdale, NJ: Erlbaum.

Hintziman, D. L., \& Rogers, M. K. (1973). Spacing effects in picture memory. Memory \& Cognition, 1, 430-434.

Hintzman, D. L., Summens, J. J., \& Block, R. A. (1975). What causes the spacing effect? Some effects of repetition, duration, and spacing on memory for pictures. Memory \& Cognition, 3 , 287-294.

Hintzman, D. L., Summers, J. J., Eki, N. T., \& Moore, M. D. (1975). Voluntary attention and the spacing effect. Memory \& Cognition, 3, 576-580.

Jensen, T. D., \& Freund, J. S. (1981). Persistence of the spacing effect in incidental free recall: The effect of external list comparisons and intertask correlations. Bulletin of the Psychonomic Society, 18, 183-186.

Johnson, P. J., Warner, M. L., \& Silleroy, R. S. (1971). Factors influencing children's concept identification performance with nonpreferred relevant attributes. Journal of Experimental Child Psychology, 11, 430-441.

Kenney, T. J., Cannizzo, S. R., \& Flavell, J. H. (1967). Spontaneous and induced verbal rehearsal in a recall task. Child Development, 38, 953-966.

KRAfT, R. N., \& Jenrins, J. J. (1981). The lag effect with aurally presented passages. Bulletin of the Psychonomic Society, 17, 132-134.

Landauer, T. K. (1969). Reinforcement as consolidation. Psychological Review, 76, 82-96.

Maskarinec, A. S., \& Thompson, C. P. (1976). The withinlist distributed practice effect: Tests of the varied context and varied encoding hypothesis. Memory \& Cognition, 4, 741-746.

McFarland, C. E., Rhodes, D. D., \& FreY, T. J. (1979). Semantic-feature variability and the spacing effect. Journal of Verbal Learning and Verbal Behavior, 18, 163-172.

Melton, A. W. (1967). Repetition and retrieval from memory. Science, 158, 532.
Melton, A. W. (1970). The situation with respect to the spacing of repetitions and memory. Journal of Verbal Learning and Verbal Behavior, 9, 596-606.

Myers, N. A., \& Perlmutter, M. (1978). Memory in the years from two to five. In P. A. Ornstein (Ed.), Memory development in children. Hillsdale, NJ: Erlbaum.

Ornstein, P. A., Naus, M. J., \& Liberty, C. (1975). Rehearsal and organizational processes in children's memory. Child Development, 26, 818-830.

Ornstein, P. A., Naus, M. J., \& Stone, B. P. (1977). Rehearsal training and developmental differences in memory. Developmental Psychology, 13, 15-24.

PAr1s, S. G. (1978). The development of inference and transformation as memory operations. In P. A. Ornstein (Ed.), Memory development in children. Hillsdale, NJ: Erlbaum.

Peterson, L. R. (1966). Short-term verbal memory and learning. Psychological Review, 73, 193-207.

Pick, A. D., Christy, M. D., \& Frankel, G. W. (1972). A developmental study of visual selective attention. Journal of Experimental Child Psychology, 14, 165-175.

Pick, A. D., \& Frankez, G. W. (1974). A developmental study of strategies of visual selectivity. Child Development, 45, $1162-1165$.

Rundus, D. (1971). Analysis of rehearsal processes in free recall. Journal of Experimental Psychology, 89, 63-77.

Shaughnessy, J. J. (1976). Persistence of the spacing effect in free recall under varying incidental learning conditions. Memory \& Cognition, 4, 369-377.

Toppino, T. C., \& DEMesquita, M. (in press). Effects of spacing repetitions on children's memory. Journal of Experimentai Child Psychology.

Toppino, T. C., Lee, N. D., Johnson, P. J., \& Shishko, S. A. (1979). Effect of perceptual pretraining on children's concep performance with nonpreferred relevant dimensions: Evidence for the role of attentional strategies. Developmental Psychol. ogy, 15, 190-196.

UNDERWOOD, B. J. (1969). Some correlates of item repetition ir free-recall learning. Journal of Verbal Learning and Verbal Be. havior, 8, 83-94.

Werner, J. S., \& Perlmutter, M. (1979). Development of vi. sual memory in infants. In H. W. Reese \& L. P. Lipsitt (Eds.) Advances in child development and behavior (Vol. 14). Nen York: Academic Press.

WiLson, W. P. (1976). Developmental changes in the lag effect: An encoding hypothesis for repeated word recall. Journal of Ex perimental Child Psychology, 22, 113-122.

\section{NOTES}

1. A $2 \times 2$ mixed analysis of variance involving grade leve (first-grade vs. preschool) and the spacing between repetition (massed vs. spaced) indicated that these variables interacted sig nificantly $[\mathrm{F}(1,34)=4.65, \mathrm{MSe}=365.60, \mathrm{p}<.05]$, thus $\mathrm{co}$ ] roborating the results of the planned comparisons.

2. The difference between predicted and obtained values $i$ probably underestimated, because the proportion of once presented items recalled almost surely was constrained by floor effect.

3. For first-graders, the estimated maximum level of reca of repeated items, assuming that repetitions were encoded ind pendently, was .373 . This was reliably lower than the obtaine level of recall for spaced repetitions $[t(17)=3.67, p<.01]$

(Manuscript received May 27, 1983; revision accepted for publication November 11,1983 .) 